\title{
Maximal Margin Local Preserving Median Fisher Discriminant Analysis for Face Recognition
}

\author{
Xingzhu Liang*, Yu'e Lin \\ School of Computer Science and Engineering, Anhui University of Science and Technology, Huainan, China. \\ * Corresponding author. Tel.: 15955460060; email: lxz9117@126.com \\ Manuscript submitted July 6, 2016; accepted October 12, 2016. \\ doi: 10.17706/jsw.11.12.1172-1181
}

\begin{abstract}
Median Fisher Discriminator(MFD) used the class median vector is more effective than Linear discriminant analysis(LDA). However, MFD only captures global geometrical structure information of the data and ignores the geometrical structure information of local data point. In this paper, we introduce a linear approach, called Maximal Margin Local Preserving Median Fisher Discriminant Analysis (MMLPMFDA). MMLPMFDA models the geometrical structure and variability of the local neighborhoods by constructing two adjacency graphs over the training data, and then incorporates the geometry and variability into the objective function of the MFD. In order to solve the small sample size problem, the objective function in a form of the difference is adopted. Finally, experiments on the ORL, YALE and AR face databases show the effectiveness of the proposed approach.
\end{abstract}

Key words: Global, local, median fisher discriminator, the small sample size problem.

\section{Introduction}

Face recognition has attracted many researchers in the area of pattern recognition, machine learning, and computer vision because of its immense application potential over the past last decades. Dimensionality reduction plays crucial role in the face recognition problem. It is generally applied for improving robustness and reducing computational complexity of the face recognition problem. Linear discriminant analysis (LDA)[1], which has been successfully applied to face recognition, is a well-known technique for dimensionality reduction. LDA searches for a representation with the restriction that the ratio of the trace of the between-class scatter and the trace of the within-class scatter is maximized when the given data are linearly distributed. However, by employing several given practical samples, the class sample average is not enough to estimate the class average accurately in particular when there are outliers in the images with noise and occlusion sample sets [2]. Thus, the accuracy of its estimate must have a substantial effect on the resulting projection directions of LDA. To overcome the weakness of the current LDA models, Yang et al. [3] proposed Median Fisher Discriminator(MFD) used the class median vector, rather than the class sample average, to estimate the class population mean vector. However, MFD only captures global geometrical structure information of the data. When MFD is applied to face recognition, it fails to discover the intrinsic manifold structure of the data, if the face images lie on a nonlinear sub-manifold hidden in the image space. Furthermore, MFD meets the small sample size problems because the median within-class matrix is singular. 
Recent studies [4]-[6] have shown that large volumes of high dimensional data possibly reside on a nonlinear sub-manifold. The two methods LDA and MFA may fail to discover the intrinsic manifold structure of the data since they can see only the global linear Euclidean structure of the data. In order to discover the intrinsic structure of the manifold, many manifold-based learning algorithms have been developed. Among them, the most representative one is Locality Preserving Projections (LPP) [4], [5]. LPP can preserves the intrinsic geometry of data and yields an explicit linear mapping suitable for training and testing samples. However, the objective function of LPP has no direct relationship to classification. In some applications, this criterion could not make sure to generate a set of good projections for classification purpose.To address the problem of "overlearning of locality", Yang et al. developed an unsupervised discriminant projection (UDP) algorithm [6] based on the idea of classification-oriented multimanifold learning. UDP characterizes the local scatter as well as the nonlocal scatter, seeking for a projection that simultaneously maximizes the nonlocal scatter and minimizes the local scatter. This property contributes to make UDP more intuitive and more powerful than most up-to-date methods. However, it must be noted that UDP is a linear approximation to the manifold learning approaches without taking the class information into account. Although LPP and UDP are effective in many domains, they are unsupervised and their unsupervised nature restrict their discriminating capability.

Motivated by LPP, many local discriminant approaches [7]-[11] have been developed for image classification, among which the most prevalent ones include margin fisher analysis (MFA)[7], locality preserving discriminant projection (LPDP)[8], local fisher discriminant analysis (LFDA)[9], local discriminant embedding(LDE)[10], locality sensitive discriminant analysis (LSDA) [11]. These methods are simple to compute and easy to operate. Several orthogonal manifold learning algorithms [12]-[14] were proposed to pursuit orthogonal bases which are believed to preserve the metric structure of the original vector space and have more locality preserving power and discriminating power.

However, a common feature of all these manifold-based learning algorithms is that they only pay attention on the local similarity information and neglect the diversity information of the sample. Impairing the diversity information of the sample may arise the over learning problem. The variation among nearby data characterizes the most important modes of variability of patterns, which will help to improve the stableness of the algorithm. Recently, From the perspective of statistic, Gao et al. introduced the diversity information between two points and proposed S-LSDP [15]. S-LSDP overcomes the over learning problem successfully and has more powerful discriminating ability.

However, the structure of real-world data is often complex and a single characterization (global or local, similarity or variation) may not be sufficient to represent the underlying intrinsic structure of data. Motivated by the success of MFD and S-LSDP in pattern regression and classification tasks, we propose a new algorithm, called Maximal Margin Local Preserving Median Fisher Discriminant Analysis or MMLPMFDA for short. The objective function of MMLPMFDA is constructed from three aspects: 1) characterizing median intra-class compactness and median inter-class separability of data points; 2)preserving the similarity of the same class and the local pattern variations;(3) using maximum scatter difference objective function [16], [17] without computing the inverse of the median within-class scatter to overcome the small size problem. The effectiveness of the proposed algorithm is demonstrated by experiments on the ORL, YALE and AR face database. The rest of this paper is organized as follows. Section 2 briefly gives an overview of related works about FDA and MFD. In Section 3, the dimensionality reduction method of MMLPMFDA is presented. In Section 4, the performance of MMLPMFDA is experimentally evaluated on two benchmark face-image databases, ORL and YALE. At last, a brief conclusion is offered in Section 5 . 


\section{Related Work}

In this section, we briefly review LDA and MFD methods for face recognition. Assume that $X$ is a $n$ dimensional face sample set with $N$ elements belonging to $C$ classes. Denote $x_{j}^{i}$ the $j$ th image in the class $i$, and $N_{i}$ number sample of the class $i$.Thus, we have $\sum_{i=1}^{C} n_{i}=N$ and $X=\left[x_{1}^{1}, x_{2}^{1}, \cdots x_{N_{C}}^{C}\right]$. The between-class scatter matrix $S_{b}$ and the within-class scatter matrix $S_{w}$ are defined as follows:

$$
\begin{aligned}
& S_{b}=\frac{1}{n} \sum_{i=1}^{C} n_{i}\left(m_{i}-m\right)\left(m_{i}-m\right)^{T} \\
& S_{w}=\frac{1}{n} \sum_{i=1}^{C} \sum_{j=1}^{n_{i}}\left(x_{j}^{i}-m_{i}\right)\left(x_{j}^{i}-m_{i}\right)^{T}
\end{aligned}
$$

where $m=\frac{1}{n} \sum_{i=1}^{C} \sum_{j=1}^{n_{i}} x_{j}^{i}$ is the mean of all the samples, and $m_{i}=\frac{1}{n_{i}} \sum_{j=1}^{n_{i}} x_{j}^{i}$ is the mean of the class $i$.

\subsection{Linear Discriminant Analysis (LDA)}

The purpose of LDA is to seek a set of projection vectors such that the fisher criterion(i.e. The ratio of the between-class scatter to the within-class scatter) is maximized after projection of samples. The criteria of LDA is defined as the following optimization problem:

$$
J(w)=\max \frac{w^{T} S_{b} w}{w^{T} S_{w} w}
$$

It has been proved that if $S_{w}$ is nonsingular, Eq.(3) can be solved by generalized eigenvalue decomposition:

$$
S_{b} w=\lambda S_{w} w
$$

\subsection{Median Fisher Discriminator (MFD)}

From Eq. (3), we can see that the class population means vector plays a central role in the definitions of the between-class and within-class scatter matrices. Thus, the accuracy of its estimate must have a substantial effect on the resulting projection directions of LDA. Since face recognition is typically a small sample size problem in which only a few of the image samples are available for training per class, it is difficult to give an accurate estimate of the class population mean using the class sample average, in particular when there are outliers in the sample set. An inaccurate estimate of the class population mean must have a negative effect on the robustness of LDA models. To overcome the weakness of the current LDA models, Median Fisher Discriminator(MFD) use the class median vector, rather than the class sample average, to estimate the class population mean vector. Median is the middle value in a distribution for a finite list of numbers. In mathematics, median refers to the number that is located at the middle of a set of numbers that have been arranged in a descending order. Denote $\bar{m}_{i}=\operatorname{median}\left(X_{i}\right)$ the middle value of the class $i$ and $X_{i}=\left[x_{1}^{i}, \cdots, x_{n_{i}}^{i}\right]=\left[\begin{array}{cccc}x_{1,1}^{i} & x_{2,1}^{i} & \cdots & x_{n_{i}, 1}^{i} \\ x_{1,2}^{i} & x_{2,2}^{i} & \cdots & x_{n_{i}, 2}^{i} \\ \vdots & \vdots & \vdots & \vdots \\ x_{1, n}^{i} & x_{1, n}^{i} & \cdots & x_{n_{i}, n}^{i}\end{array}\right]$. The symbol median(.) is the median operator 
of a set numbers. Then, $\bar{m}_{i, j}=\operatorname{median}\left(x_{1, j}^{i}, x_{2, j}^{i} \cdots, x_{n_{i}, j}^{i}\right)$ is the median of elements on the $j$ th row of the $\bar{m}_{i}$. Denote $x_{1, j}^{i}>x_{2, j}^{i}>\cdots>x_{n_{i}, j}^{i}$ arranged in a descending order. The $\bar{m}_{i, j}$ is computed as follow:

$$
\bar{m}_{i, j}=\left\{\begin{array}{cc}
x_{\left(n_{i}+1\right) / 2, j}^{i} & \text { if } n_{i} \text { is odd number } \\
\left(x_{n_{i} / 2, j}^{i}+x_{n_{i} / 2+1, j}^{i}\right) / 2 & \text { if } n_{i} \text { is even number }
\end{array}\right.
$$

Then, the between-class scatter matrix $S_{b}$ is redefined as:

$$
\bar{S}_{b}=\frac{1}{n} \sum_{i=1}^{C} n_{i}\left(\bar{m}_{i}-m\right)\left(\bar{m}_{i}-m\right)^{T}
$$

where $\bar{m}_{i}=\operatorname{median}\left(x_{1}^{i}, x_{2}^{i}, \cdots, x_{n_{i}}^{i}\right)$ is the median mean of the class $i$. In order to discuss following new method, $\bar{S}_{b}$ is called the median between-class scatter matrix.

The within-class scatter matrix $S_{w}$ is redefined as:

$$
\bar{S}_{w}=\frac{1}{n} \sum_{i=1}^{C} \sum_{j=1}^{n_{i}} w_{i j}\left(x_{j}^{i}-\bar{m}_{i}\right)\left(x_{j}^{i}-\bar{m}_{i}\right)^{T}
$$

where $\bar{S}_{w}$ is called the median within-class scatter, and $w_{i j}$ is the weighting coefficient. This weight is introduced to alleviate the influence of outliers on the construction of the within-class scatter matrix. The $w_{i j}$ is defined as:

$$
w_{i j}=\exp \left(-\frac{\left\|x_{j}^{i}-m_{i}\right\|^{2}}{t}\right)
$$

where $t$ is a parameter, and $t \in(0,+\infty)$. The criteria of MFD is defined as the following optimization problem:

$$
J(w)=\max \frac{w^{T} \bar{S}_{b} w}{w^{T} \bar{S}_{w} w}
$$

Eq.(8) can be solved by generalized eigenvalue decomposition:

$$
\bar{S}_{b} w=\lambda \bar{S}_{w} w
$$

\section{Maximal Margin Local Preserving Median Fisher Discriminant Analysis}

We propose a novel efficient approach, called Maximal Margin Local Preserving Median Fisher Discriminant Analysis (MMLPMFDA). In order to preserve the intrinsic manifold structure of the data, the MMLPMFDA attempt to learn a linear transformation that could deal with the similarity and diversity information simultaneously. MMMDFDA considers preserving the similarity of the same class and variation among nearby data points. Therefore, two weighted matrix are constructed: weighted adjacency similarity matrix $S$ and weighted adjacency diversity matrix $B$. The former, constructed using the class information, measures the similarity of the points and the latter measures the diversity of the local points. $S$ is a weighted matrix with elements characterizing the similarity of two points and $B$ is a weighted matrix with the elements characterizing the diversity of two points.

The elements of similarity weighted matrix $S$ is defined as following: 


$$
S_{i j}= \begin{cases}\exp \left(-\frac{\left\|x_{i}-x_{j}\right\|^{2}}{t}\right) & \text { if } x_{i} \text { is among } k_{1} \text { nearest neighbors of } x_{j} \text { or } \\ 0 & x_{j} \text { is among } k_{1} \text { nearest neighbors of } x_{i} \text { and } l_{\mathrm{i}}=l_{\mathrm{j}} \\ & \text { otherwise }\end{cases}
$$

where $t \in(0,+\infty)$ and $l_{i}$ denotes the class label of $x_{i}$. The elements of diversity weighted matrix $B$ is defined as following:

$$
B_{i j}= \begin{cases}\exp \left(\frac{-b}{\left\|x_{i}-x_{j}\right\|^{2}}\right) & \text { if } x_{i} \text { is among } k_{1} \text { nearest neighbors of } x_{j} \text { or } \\ 0 & x_{j} \text { is among } k_{1} \text { nearest neighbors of } x_{i} \\ & \text { otherwise }\end{cases}
$$

where $b \in(0,+\infty)$.

Let $S_{L}$ denote the local similarity scatter matrix, and $S_{N}$ denote the local variations scatter matrix defined as follows, respectively:

$$
\begin{aligned}
& S_{L}=\frac{1}{2 N N} \sum_{i=1}^{N} \sum_{j=1}^{N} S_{i j}\left(x_{i}-x_{j}\right)\left(x_{i}-x_{j}\right)^{T} \\
& S_{N}=\frac{1}{2 N N} \sum_{i=1}^{N} \sum_{j=1}^{N} B_{i j}\left(x_{i}-x_{j}\right)\left(x_{i}-x_{j}\right)^{T}
\end{aligned}
$$

In order to preserve the local similarity information of the same class and variation among nearby data points, we impose the following objective:

$$
J(w)=\max \frac{w^{T} S_{N} w}{w^{T} S_{L} w}
$$

As aforementioned analysis, the objective functions Eq.(9) and Eq.(15) characterize the global discriminant information, the similarity information of the data points from the same class and the local pattern variations, respectively. If we want all these two objective functions to be satisfied simultaneously, a suitable discriminant feature extraction criterion should be in the form as follows:

$$
J(W)=\max \frac{W^{T}\left[\alpha \bar{S}_{b}+(1-a) S_{N}\right] W}{W^{T}\left[\varepsilon \bar{S}_{w}+(1-\varepsilon) S_{L}\right] W}
$$

where $0<a<1$ and $0<\varepsilon<1$ are non-negative constants to balance the two terms of the objective function. In order to avoid the small sample size problem, we change the objective function into a form of difference

$$
J(W)=\max W^{T}\left\{\left[\alpha \bar{S}_{b}+(1-a) S_{N}\right]-\left[\varepsilon \bar{S}_{w}+(1-\varepsilon) S_{L}\right]\right\} W
$$

To eliminate the freedom that we can multiply $\mathrm{W}$ with some nonzero scalar, we add the constraint

$$
W^{T} W=I
$$

where $I$ is an identity matrix. Thus the goal of MMMDFDA algorithm is just to solve the following optimization problem: 


$$
\begin{array}{ll}
J(W)=\max & W^{T}\left\{\left[\alpha \bar{S}_{b}+(1-a) S_{N}\right]-\left[\varepsilon \bar{S}_{w}+(1-\varepsilon) S_{L}\right]\right\} W \\
& \text { s.t. } W^{T} W=I
\end{array}
$$

Eq.(19) can be solved by generalized eigenvalue decomposition:

$$
\left\{\left[\alpha \bar{S}_{b}+(1-a) S_{N}\right]-\left[\varepsilon \bar{S}_{w}+(1-\varepsilon) S_{L}\right]\right\} w=\lambda w
$$

\section{Experimental Results}

In this section we will carry out a set of experiments to show the effectiveness of our method for face recognition. We employ three widely used face databases (ORL, YALE and AR) to evaluate the performance of MMMDFDA and compare it with two dimensionality reduction methods including MFD and S-LSDP. In classification stage, we use the Euclidean metric to measure the dissimilarity between two feature vectors and the nearest classifier for classification because of its simplicity. The parameters for MMMDFDA are set as $a=0.5$ and $\beta=0.5$.

The ORL face database [18] contains 400 face images of 40 distinct subjects. For some subjects, the images were taken at different times, varying the lighting, facial expressions (open/closed eyes, smiling/not smiling) and facial details (glasses/no glasses). All the images were taken against a dark homogeneous background with the subjects in an upright, frontal position (with tolerance for some side movement). All the images were in grayscale and resized to $64 \times 64$ pixel. Fig. 1 shows the sample images of one person.

The YALE face database [19] contains 165 gray scale images of 15 individuals, each individual has 11 images. The images demonstrate variations in lighting condition, facial expression (normal, happy, sad, sleepy, surprised, and wink). All the images were in grayscale and resized to $64 \times 64$ pixel. Fig. 2 shows the sample images of one person.

The AR face database [20] contains over 4000 color face images from 126 people, including frontal views of faces with different facial expressions, lighting conditions, and occlusions. In the experiments,we choose a subset of AR database.The subset of AR database contains 100 individuals and 10 images for person. All the images were in grayscale and resized to $100 \times 80$ pixel. Fig. 3 shows the sample images of one person.

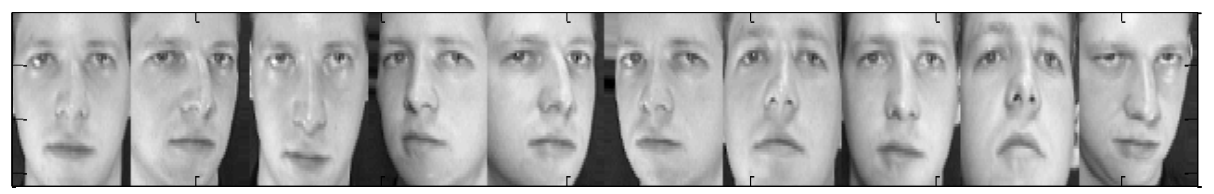

Fig. 1. Images of one person in ORL database.

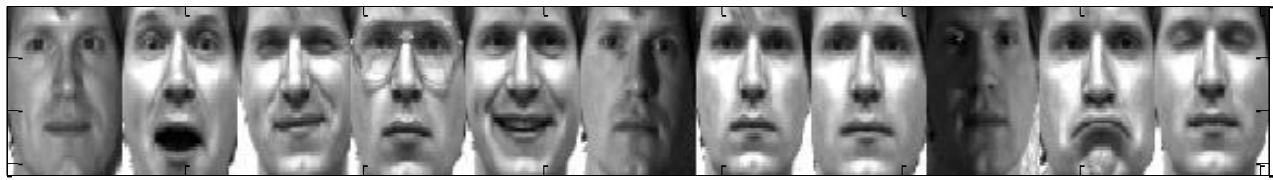

Fig. 2. Images of one person in YALE database.

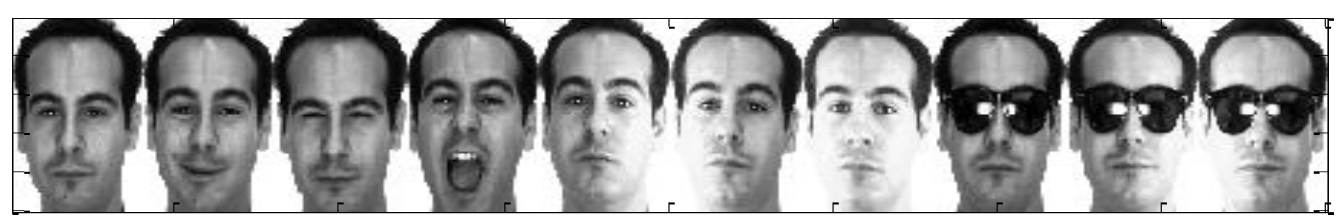

Fig. 3. Images of one person in AR database. 
In order to evaluate the performance of our propose method, the first 5 images per person are selected as training, respectively, and the corresponding rest images for testing. Thus, the performance curves for the three methods are shown in Fig. 4, 5 and 6.

Fig. 4, 5 and 6 show the recognition rates of MMLPMFDA, MFD and S-LSDP with respect to the number of the discriminant vectors. When the number of training samples of each person is increasing, S-LSDP, MFD and MMLPMFDA nearly have better recognition rates. Fig. 4, 5 and 6 indicate that with the increasing of the dimensions, our proposed method outperforms others. The main reason may be that our method could deal with both the global and local information, and the strong structure preserving and discriminating ability make the method more suitable for classification.

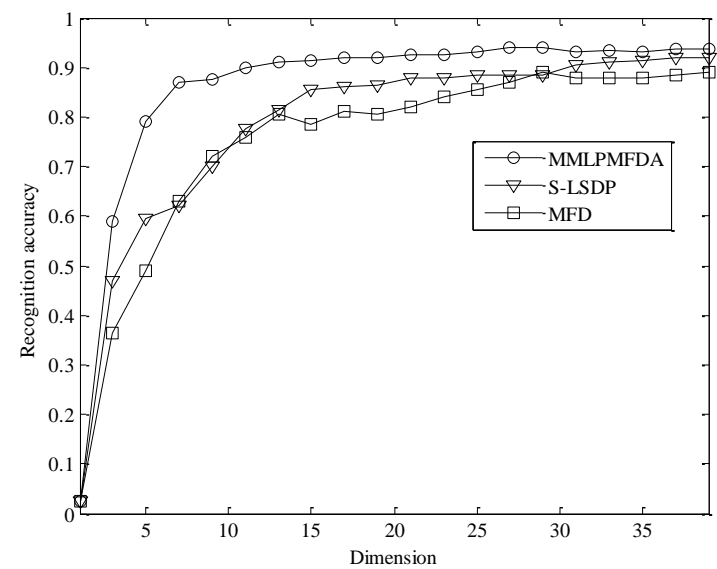

Fig. 4. Recognition rate curves for different algorithms with varied dimensions (in ORL database).

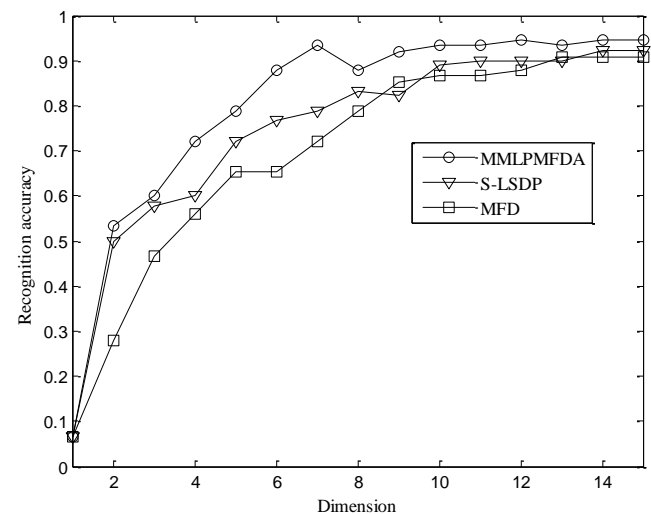

Fig. 5. Recognition rate curves for different algorithms with varied dimensions (in YALE database).

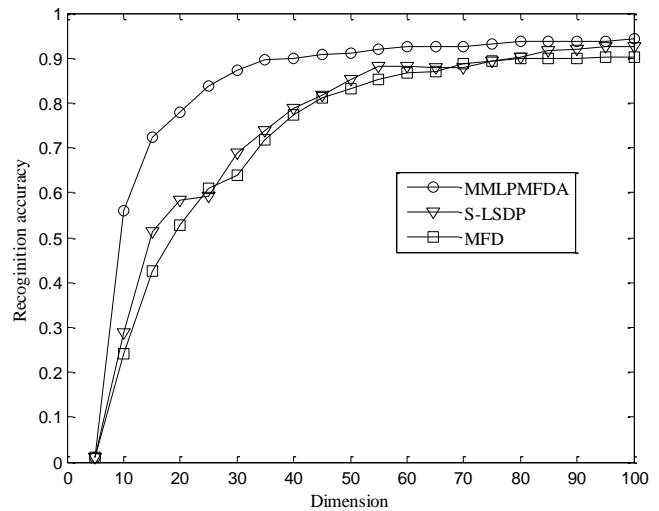

Fig. 6. Recognition rate curves for different algorithms with varied dimensions (in AR database). 
We also randomly select the $p$ images ( $p$ varying from 3 to 7 ) from each person as training set and the rest images as testing set. The recognition rate for the three methods are listed in Table 1,2 and 3 .

Table 1. The Comparison of Recognition Rates(\%) Using MMLPMFDA, MFD and S-LSDP with Different Number of Training Samples on ORL Database

\begin{tabular}{cccccc}
\hline \hline methods & 3 & 4 & 5 & 6 & 7 \\
\hline MMLPMFDA & 90.71 & 92.50 & 93.75 & 95.63 & 97.33 \\
MFD & 89.29 & 91.67 & 92.25 & 93.13 & 95.00 \\
S-LSDP & 90.00 & 92.08 & 93.00 & 93.75 & 95.83 \\
\hline \hline
\end{tabular}

Table 2. The Comparison of Recognition Rates(\%) Using MMLPMFDA, MFD and S-LSDP with Different Number of Training Samples on YALE Database

\begin{tabular}{cccccc}
\hline \hline methods & 3 & 4 & 5 & 6 & 7 \\
\hline MMLPMFDA & 87.50 & 91.42 & 94.44 & 96.00 & 96.67 \\
MFD & 85.00 & 88.57 & 92.22 & 93.33 & 94.26 \\
S-LSDP & 85.83 & 89.52 & 92.22 & 94.66 & 95.15 \\
\hline \hline
\end{tabular}

Table 3. The Comparison of Recognition rates(\%) using MMLPMFDA, MFD and S-LSDP with Different Number of Training Samples on AR Database

\begin{tabular}{cccccc}
\hline \hline methods & 3 & 4 & 5 & 6 & 7 \\
\hline MMLPMFDA & 92.57 & 94.16 & 95.45 & 95.25 & 96.33 \\
MFD & 91.42 & 93.00 & 93.20 & 93.75 & 94.66 \\
S-LSDP & 91.71 & 93.66 & 94.00 & 94.50 & 95.66 \\
\hline \hline
\end{tabular}

From Table 1, 2 and 3 we can see that the maximal recognition rate of the MMLPMFDA increase with the increasing of the train number. The one reason may be that the median intra-class compactness and the median inter-class are used in the MMLPMFDA modelling. The other reason may be that our method can deal with the similarity and diversity information simultaneously. Furthermore, the strong structure preserving and discriminating ability make the proposed method more suitable for the recognition tasks. MFD fails to explore the intrinsic structure of the data, so it always performed the worst. In addition, from a practical point of view, MMLPMFDA offers a stable algorithm to handle recognition tasks.

\section{Conclusions}

In this paper, we propose a novel supervised algorithm, namely Maximal Margin Local Preserving Median Fisher Discriminant Analysis(MMLPMFDA). The median intra-class compactness and the median inter-class are used in the MMLPMFDA modelling, which is more robust to special circumstances such as noise, bright light, some outliers will appear in the practical input databases. On the other hand, MMLPMFDA can effectively preserve the similarity of the same class and the local pattern variations, which can find more discriminating features for face recognition. Using maximum scatter difference method without computing the inverse of the median within-class scatter, MMLPMFDA does not suffer from the SSS problem. Experimental results show the effectiveness of the proposed method and its potential with respect to MFD method and ELDA.

In the future, the following work will be interesting to us: (i) focusing on the sparsity classification methods[21,22], new manifold learning classification algorithms can be developed to enhance their 
performances in face recognition; (ii) focusing on the incremental learning methods [23,24], the incremental feature set could be utilized by learning classification algorithms to improve their processing speed for real-time classification and recognition.

\section{Acknowledgement}

This article is supported by the Key Project of Higher Education Natural Science Foundation of Anhui Province (No. KJ2016A203) and the Master and Doctor Foundation of Anhui University of Science and Technology(No. 2010yb026).

\section{References}

[1] Belhumeur, P. N., Hespanha, J. P., \& Kriegman, D. J. (1997). Eigenfaces vs fisherfaces: Recognition using class specific linear projection. IEEE Transactionson Pattern Analysis and Machine Intelligence, 19(7), 711-720.

[2] Yang, J., Zhang, D., \& Yang, J. Y. (2006). Median LDA: A robust feature extraction method for face recognition. Proceedings of the IEEE International Conference on Systems, Man, and Cybernetics (pp. 4208-4213)

[3] Yang, J., Yang, J. Y., \& Zhang, D. (2008). Median fisher discriminator: A robust feature extraction method with applications to biometrics. Frontiers of Computer Science in China, 2(3), 295-305.

[4] He, X. F., Yan, S. C., Hu, Y. et al. (2005). Face recognition using Laplacianfaces. IEEE Transactions on Pattern Analysis and Machine Intelligence, 27(3), 328-340.

[5] He, X. F. N. (2004). Locality preserving projections. Proceedings of Advances in Neural Information Processing Systems 16 (pp. 153-160). MA: Cambridge. MIT Press.

[6] Yang, J., Zhang, D., Yang, J. Y. et al. (2007). Globally maximizing, locally minimizing: unsupervised discriminant projection with application to face and palm biometrics. IEEE Trans Pattern Anal Mach Intell, 29(4), 650-664.

[7] Xu, D., Yan, S., Tao, D., et al. (2007). Marginal fisher analysis and its variants for human gait recognition and content-based image retrieval. IEEE Transactions on Image Processing, 16(11), 2811-2821.

[8] Gui, J., Jia, W., Zhu, L. et al., (2010). Locality preserving discriminant projection for face and palmprint recognition. Neurocomputing, 73(13-15), 2696-2707.

[9] Sugiyama, M. (2007). Dimensionality reduction of multimodal labeled data by local fisher discriminant analysis, Journal of Machine Learning Research, 1027-1061.

[10] Chen, H. T., Chang, H. W., \& Liu, T. L. (2005). Local discriminant embedding and its variants. Proceedings of the IEEE Computer Visual and Pattern Recognition (pp. 846-853)

[11] Cai, D., He, X., Zhou, K. et al., (2007). Locality sensitive discriminant analysis. Proceedings of the 20th International Joint Conference Artificial Intelligence (pp. 708-713)

[12] Zhu, L., \& Zhu. S. (2007). Face recognition based on orthogonal discriminant locality preserving projections, Neurocomputing, 70(9), 1543-1546.

[13] Hu, H. F. (2008). Orthogonal neighborhood preserving discriminant analysis for face recognition. Pattern Recognition, 41(6), 2045-2054.

[14] Lei, Y. K., Zou, J. W., Dong, T. B. et al., (2014). Orthogonal locally discriminant spline embedding for plant leaf recognition. Computer Vision and Image Understanding, 119(2), 116-126.

[15] Gao, Q. X., Xie, D. Y., Xiu, H., et al. (2010). Supervised feature extraction based on information fusion of local structure and diversity information. Acta Autom. Sin, 36(8), 1108-1114.

[16] Li, H., Jiang, T., \& Zhang, K. (2006). Efficient and robust feature extraction by maximum margin criterion, IEEE Transactions on Neural Network, 17(1), 157-165. 
[17] Chen, X., \& Zhang, J. S. (2012). A novel maximum margin neighborhood preserving embedding for face recognition. Future Generation Computer Systems, 28(1), 212-217.

[18] The ORL Face Database. Retrieved from http://www.uk.research.att.com/facedatabase.html

[19] Yale Univ Face Database. Retrieved from http://cvc.yale.edu/projects/yalefaces/yalefaces.html

[20] The AR face database. Retrieved from http://rvl1.ecn.purdue.edu/aleix/aleixfaceH_DB.html

[21] Wang, S. J., Yang, J., Sun, M. F. et al., (2013). Sparse tensor discriminant color space for face verification, IEEE Transactions on Neural Networks and Learning Systems, 23(6), 876-888.

[22] Gu, Z. H., \& Yang, J. (2013). Sparse margin-based discriminant analysis for feature extraction. Neural Computing and Applications, 23(6), 1523-1529.

[23] Wan, J. G., Sung, E., \& Yau, W. Y. (2010). Incremental two-dimensional linear discriminant analysis with applications to facerecognition. Neurocomputing, 33(3), 314-322.

[24] Choi, Y., Ozawa, S., \& Lee, M. (2014). Incremental two-dimensional kernel principal component analysis. Neurocomputing, 134(6), 280-288.

Xing-Zhu Liang was born in Anhui, China, in 1979. He received the MS degree in computer science at Daqing Petroleum Institute, China, in 2006. Now, he is a lecturer at Anhui University of Science \& Technology in Huainan city, China. His research interests include computer vision, computer network security, and pattern recognition.

Yu-E Lin was born in Heilongjiang, China, in 1979. She received the MS degree in computer science at Daqing Petroleum Institute, China, in 2006. She received the PhD degree in computer science at Harbin Engineering University, China, in 2009. She is currently an associate professor at Anhui University of Science \& Technology in Huainan city, China. Her research interests are in the areas of pattern recognition, image processing, and artificial intelligence. 